\title{
Eine Analyse der individuellen Wahrnehmungs- und Bewältigungsstrategien von Menschen mit Demenz im Frühstadium ihrer Erkrankung unter Beachtung der Funktion und Wirksamkeit von Selbsthilfegruppen auf der Grundlage von Selbstäußerungen
}

- Was ist (zu dieser Thematik) schon bekannt?

Selbstäußerungen von Menschen mit Demenz im Frühstadium ihrer Erkrankung zu analysieren, stellen eine wichtige Quelle dar, um ihre Bedürfnisse und Bedarfe zu erfassen.

Was ist neu? Menschen mit Demenz, die sich in Selbsthilfegruppen engagieren, werden darüber befragt, wie sie mit ihrer Erkrankung leben und welche Funktion eine Selbsthilfegruppe in der Bewältigung derselben hat.

- Welche Konsequenzen haben die Ergebnisse für die Pflegepraxis? In Bezug auf Beratungs- und Unterstützungsangebote für Menschen mit Demenz in einer frühen Phase ihrer Erkrankung ist darauf zu achten, das labile System zwischen Hilfsangeboten und Fürsorge so zu reflektieren, dass Entmündigungsprozesse minimiert werden.

Das gesellschaftliche Bild eines Menschen mit Demenz im Frühstadium der Erkrankung, ist getragen von einem impliziten Widerspruch: es handelt sich um Menschen, die im Prinzip (noch) selbstbestimmt denken und handeln können, die aber genau dabei Hilfe von außen benötigen. Selbsthilfegruppen stellen eine mögliche Form der Unterstützung dar. Es ist die Frage zu beantworten, wie sich Menschen mit Demenz im Frühstadium ihrer selbst wahrnehmen und welchen Einfluss die Intervention einer Selbsthilfegruppe auf ihr Selbstkonzept hat. Es wurden bisher insgesamt fünf Gruppendiskussionen und acht narrative biografisch orientierte Interviews durchgeführt. Folgende Ergebnisse für Menschen mit Demenz sind festzuhalten: Sie sehen sich Entmündigungsprozessen durch Menschen in ihrem sozialen Umfeld ausgesetzt. In den Selbsthilfegruppen werden demgegenüber Prozesse der Anerkennung der eigenen Person als chronisch kranker Mensch gefördert. Zwei Schlussfolgerungen für den Umgang mit Menschen mit Demenz und die Ausgestaltung von Hilfestrukturen sind $z u$ ziehen: Formen der Entmündigung von außen gilt es abzubauen. Selbsthilfegruppen in einer unterstützenden Funktion sollten dementsprechend die bisherige Angebotsstruktur für Menschen mit Demenz ergänzen.

Schlüsselwörter: Menschen mit Demenz, Selbsthilfegruppe, Intervention
Problem- und Fragestellung

Das Bild eines Menschen mit Demenz im Frühstadium der Erkrankung ${ }^{1}$ beginnt sich in den letzten Jahren auch in Deutschland $\mathrm{zu}$ wandeln. Von einer eher defizitorientierten Sicht, die auf eine Desintegration von Menschen mit Demenz fokussiert, rücken zunehmend mehr ressourcenorientierte Aspekte in den Mittelpunkt. Sie deuten eine gesellschaftliche Integration an (Altimisik, 2009; Hauser, 2006; Hauser, 2009; Lakotta, 2010a, 2010b, 2010c; Langehennig \& Obermann, 2006; Niebuhr, 2004; Sowarka, 2008; Demenz Support Stuttgart, 2010a, 2010b, 2010c; Demenz Support Stuttgart, 2012; Rohra, 2011). Menschen mit Demenz im Frühstadium ihrer Erkrankung in der Bewältigung ihrer Krankheit ressourcenorientiert und individuell passgenau zu unterstützen, bedingt in einem ersten Schritt die Beantwortung der Frage, welche Bedürfnisse und Bedarfe diese Menschen aus ihrer Sicht eigentlich haben. In den letzten Jahren

\footnotetext{
1 Es wird im Rahmen der Studie keine Auswah der Interviewpartner(innen) entlang des Kriteriums der Demenzformen vorgenommen. Die Begrenzung auf ein Frühstadium der Erkrankung ergibt sich aus der gewählten Untersuchungsgruppe selbst. Die Teilnahme von Menschen mit Demenz an einer Selbsthilfegruppe setzt eine gewisse kognitive Fähigkeit und Bereitschaft zur Kommunikation voraus. Wenn diese nicht mehr vorhanden ist, wird nach Aussagen der Beteiligten aus einer Selbsthilfegruppe eine Betreuungsgruppe.

Eine Frühphase der Demenz liegt dann vor, wenn nach ICD-10 «Gedächtnis und andere kognitive Funktionen oder Alltagsaktivitäten eingeschränkt sind, aber ein unabhängiges Leben weiter möglich ist.» (Hauser, 2009: 22). Die Behinderungen im Umgang mit dem alltäglichen Leben sind nicht einschneidend. Eine Hilfestellung ist nur bedingt erforderlich. Die Bewältigungsmechanismen sind noch weitgehend selbstgesteuert.
} 
wächst die Erkenntnis, dass man sie dazu auch selbst befragen kann und muss. Dabei wird deutlich, dass der biografische Hintergrund den individuellen Umgang mit kritischen Lebensereignissen (Filipp \& Aymanns, 2010: 13) und Bewältigungsmustern in einem hohen Maße mit beeinflusst (Griese \& Griesehop, 2007; Rosenthal, 1995; Rosenthal, 2010; Schütze, 1981; Schütze, 1983). Die Selbstwahrnehmung von Menschen mit Demenz auf der Grundlage ihrer Selbstäußerungen zu analysieren, bietet so einen sinnvollen $\mathrm{Zu}$ gang, um einen individuell passenden Unterstützungsansatz zu finden (Bell \& Troxel, 2006; De Boer et al., 2007; Goldsmith, 2005; Kutzleben et al., 2012; Niebuhr, 2004; Roest et al., 2007; Snyder, 2009; Stechl, 2006a; Steeman et al., 2006). Selbsthilfegruppen nehmen in dem Prozess der ressourcenorientierten Bewältigung von Demenz, so die Ergebnisse angloamerikanischer Studien, eine wichtige Funktion ein (LaBarge \& Tranji, 1995; Logsdon et al., 2010; Snyder et al., 2007; Yale, 1995). Auch in der Bundesrepublik Deutschland haben sich in den letzten Jahren vermehrt Selbsthilfegruppen nach dem angloamerikanischen Vorbild als Support Groups für Menschen mit Demenz gebildet. Die Rekonstruktion der individuellen Bewältigungsstrategien von Menschen mit Demenz im Frühstadium ihrer Erkrankung und die Erfassung des Einflusses, den dabei Selbsthilfegruppen einnehmen, ist ein wesentliches Ziel der Studie SEIN ${ }^{2}$

2 Vollständiger Titel der Studie: Selbstbestimmung und Intervention (SEIN). Die Analyse der Funktion und Wirksamkeit von Selbsthilfegruppen für Menschen mit Demenz im Frühstadium im Vergleich zu Menschen mit Multipler Sklerose auf der Grundlage von Selbstäußerungen. Die exemplarisch ausgewählte Vergleichsgruppe der Menschen mit Multipler Sklerose, die sich in einer Selbsthilfegruppe engagieren, wurde in die Darstellung der Teilergebnisse nicht einbezogen. Das hätte den Rahmen eines Artikels gesprengt. Der Schwerpunkt der Ausführungen liegt somit auf dem Umgang von Menschen mit Demenz mit ihrer Krankheit. gewesen. Vor diesem Hintergrund wurden Variablen erfasst, die eine Implementierung von passgenauen Interventionen vorbereiten. Im Folgenden werden Teilergebnisse aus der Studie vorgestellt. Der Fokus liegt auf der Rekonstruktion der Innensicht der Menschen mit Demenz selbst. Ihre Handlungsmodelle werden anhand von immanenten Kategorien entwickelt. Die Funktion der Selbsthilfegruppe im Bewältigungsprozess wird analysiert. Es ist die Frage zu beantworten, wie sich Menschen mit Demenz im Frühstadium ihrer Erkrankung selbst wahrnehmen und welchen Einfluss die Intervention einer Selbsthilfegruppe auf ihr Selbstkonzept hat.

\section{Das Ziel}

Ziel der folgenden Ausführungen ist es, herauszuarbeiten, wie sich Menschen mit Demenz in der Frühphase ihrer Erkrankung, die sich in Selbsthilfegruppen engagieren, selbst wahrnehmen und welche Rolle Selbsthilfegruppen in der Entwicklung des Selbstkonzeptes einnehmen, um vor diesem Hintergrund erste Empfehlungen für die Pflegepraxis geben zu können.

\section{Material und Methoden}

In der qualitativ angelegten empirischen Studie SEIN wurden vor dem Hintergrund einer Literaturanalyse, die der Konzeptentwicklung und Leitfadenkonstruktion diente, unter anderem auch Gruppendiskussionen mit Menschen mit Demenz durchgeführt, die sich in einer Selbsthilfegruppe engagieren. Sie dienten der Analyse der Funktion, die eine Selbsthilfegruppe für die Bewältigung der Erkrankung hat. Ergänzend wurden ebenfalls offene, narrativ angelegte Einzelinterviews mit Menschen mit Demenz, die an die- sen Selbsthilfegruppen teilnahmen, durchgeführt. Sie dienten der Ermittlung der biografisch geformten individuellen Bewältigungsstrategien im Umgang mit Demenz.

Die Erhebungs- und Auswertungsverfahren der Interviews und Gruppendiskussionen folgten generell den methodologischen Prinzipien der rekonstruktiven Sozialforschung (Bohnsack, 2010: 20). Das Verfahren zur Erhebung der Einzelinterviews orientierte sich an dem Grundmuster der von Schütze entwickelten Verfahren für narrative biografische Interviews, fokussierte dabei allerdings thematisch auf das Erleben der Krankheit und die Funktion, die der Selbsthilfegruppe in der Bewältigung der Krankheit zukommt (Schütze, 1983). Das Erhebungsverfahren der Gruppendiskussion orientierte sich an dem von Mannheim entwickelten und von Bohnsack aufgegriffenen Verfahren. Das Verständnis der Gruppe als «konjunktiven Erfahrungsraum» bedingte dabei eine offene, erzählgenerierende Interviewtechnik (Bohnsack, 2010: 108 ff.).

\section{Rekrutierung und Auswahl der Studienteilnehmenden}

Die Rekrutierung und Auswahl der Interviewpartner(innen) sowie der Gruppendiskussionsteilnehmenden erfolgte über den Bundesverband der Alzheimer Gesellschaft e.V. und seine und Regionalgruppen. An den Dachverband und seine Regionalgruppen wurde eine Anfrage formuliert, um Selbsthilfegruppen beziehungsweise unterstützte Selbsthilfegruppen, die unter dem jeweiligen Dachverbänden organisiert sind, zu erfassen. Es erfolgte keine regionale Begrenzung. Die entsprechenden Selbsthilfegruppen wurden angeschrieben und dann telefonisch kontaktiert, um eine mögliche Mitarbeit zu erfragen. Wenn sich aus diesen Selbsthilfegruppen einzelne Menschen bereiterklärten, ein biografi- 
sches Interview zu führen, dann wurden entsprechende Termine mit einer angemessenen Vor- und Nachbereitung geplant. Die Auswahl der Teilnehmenden folgt also einzig und allein ihrem persönlichen Interesse und ihrer Bereitschaft.

Da Selbsthilfegruppen für Menschen mit Demenz erst im Entstehen begriffen sind und $\mathrm{zu}$ dem Zeitpunkt der Erhebung auch nicht über eine eigene Vernetzungsbasis verfügten, wurde eine weitere Suchstrategie angeschlossen. Erste Informationen über existierende Selbsthilfegruppen, die durch die Alzheimer Gesellschaft e.V. und den Demenz Support Stuttgart gesammelt worden waren, wurden genutzt, um Kontaktpersonen als Zugang zu Selbsthilfegruppen zu ermitteln. Sie wurden schriftlich und bei Rückmeldung auch telefonisch kontaktiert. Mit den Selbsthilfegruppen, die ihre Bereitschaft signalisierten, wurden entsprechende Gruppendiskussionen durchgeführt. Mit den Einzelpersonen wurden entsprechende Interviewtermine $\mathrm{zu}$ der Zeit und an dem Ort abgesprochen, den sie dafür als geeignet angaben.

\section{Ein- und Ausschlusskriterien der Studienteilnehmenden}

Eingeschlossen wurden bei der Auswahl der Selbsthilfegruppen für Menschen mit Demenz alle Gruppen, die von den Betroffenen selbst beziehungsweise auch als unterstützte Selbsthilfegruppen von Nichtbetroffenen als professionelle Begleitungen im Rahmen von Gesprächskreisen durchgeführt wurden, sich in regelmäßigen Abständen trafen und über öffentliche Medien auch für Außenstehende und Neuaufnahmen erreichbar und offen waren. Ausgeschlossen wurden Selbsthilfegruppen, an denen auch Angehörige gemeinsam mit Menschen mit Demenz teilnahmen. Ausgeschlossen wurden ebenfalls solche Selbsthilfegruppen, die weniger als Gesprächs- kreise, sondern eher als Freizeit- und Sport- oder Gymnastikgruppen arbeiteten.

\section{Datenerhebung}

Als Erhebungsinstrumente im Rahmen der qualitativen empirischen Studie SEIN wurden Interviews und Gruppendiskussionen mit den Menschen mit Demenz im Frühstadium eingesetzt, die sich bereits in Selbsthilfegruppen engagieren. Die Gruppendiskussionen und Interviews wurden durchgängig von der Forschenden selbst durchgeführt, die bereits über eine hinreichend umfangreiche Forschungserfahrung verfügt. Sie wurden immer mit der gleichen offenen, erzählgenerierenden Frage eingeleitet: «Wie ist das, wenn man mit Demenz lebt und sich in einer Selbsthilfegruppe engagiert?»

Auf der Grundlage von Selbstäußerungen wurde die Eigenlogik ${ }^{3}$ der von Demenz betroffenen Menschen, ihr Blick auf die eigenen Erfahrungen und Erlebnisse rekonstruiert. Ihre Selbstwahrnehmung im Umgang mit der Demenz, ihre Bewältigungsstrategien und ihre Einschätzung, welchen Beitrag die Selbsthilfegruppe dazu leistet, galt es zu erfassen. Dazu wurde ein methodisches Verfahren der Auswertung beziehungsweise Textanalyse entwickelt, das drei methodische Verfahrensweisen kombinierte.

\section{Datenauswertung/Datenanalyse}

Die bisherige Auswertung beruht auf der qualitativen Analyse von insgesamt fünf transkribierten Gruppendiskus-

3 Der Begriff der «Eigenlogik» respektive de Selbstreferenzialität als ein zentraler Begriff aus der Luhmannschen Systemtheorie (Luhmann, 1993) verweist darauf, dass Systeme jeweils eine eigene Ordnung ausprägen. In der rekonstruktiven Sozialforschung beschreibt der Begriff der Eigenlogik für die kleinste Systemeinheit, den Menschen, eine entsprechende Fähigkeit des Menschen, sich selbst zu erklären. Eine zentrale Aufgabe, die den Umgang mit qualitativen Daten bestimmt ist es nun, diese jeweilige eigene Sinnstruktur von Individuen zu rekonstruieren. sionen (zwei Selbsthilfegruppen wurden in einem Abstand von acht Monaten ein zweites Mal befragt) und acht narrativen Interviews (die Interviewpartner(innen) wurden in einem Abstand von acht Monaten ebenfalls ein zweites Mal befragt) zwei Männer und zwei Frauen im Alter zwischen 58 und 75 Jahren.

An den Gruppendiskussionen nahmen zwischen fünf (zwei Männer und drei Frauen) und neun Personen (zwei Männer und sieben Frauen) in Selbsthilfegruppen für Menschen mit Demenz teil. Das Alter der Teilnehmenden wurde nicht erfragt. Die Dauer der Gruppendiskussionen variierte zwischen 44 und 118 Minuten. Die Dauer der Interviews betrug zwischen 46 und 90 Minuten. Die Gruppendiskussionen wurden immer im Rahmen eines regulären Treffens der Selbsthilfegruppe an dem dafür vorgesehen Ort durchgeführt. Die Gruppengröße schwankte zwischen fünf und neun Personen. Die Interviewpartner(innen) wurden entsprechend ihrem Wunsch in ihren eigenen Wohnungen interviewt. Die Auswahl der Gesprächspartner(innen) erfolgte nach dem Prinzip der Freiwilligkeit. Wichtig war nur, dass diese auch in denselben Selbsthilfegruppen engagiert waren, die in einem Gruppendiskussionsverfahren befragt worden waren. Damit ließ sich ein Vergleich zwischen der Gruppenposition und der Einzelwahrnehmung herstellen. In den Gruppendiskussionen wurde so danach gefragt, wer bereit sei, ein Einzelinterview durchzuführen. Insgesamt sechs Menschen meldeten sich, von denen letztendlich vier Personen übrig blieben. Die Anzahl erschien für eine offene narrative Interviewform ausreichend.

Die Konstruktion des Interviewleitfadens fand vor dem Hintergrund der Literaturanalyse und einer entsprechender Konzeptbildung (Panke-Kochinke, 2012) statt. Die Konzepte des 
«kritischen Lebensereignisses» (Filipp \& Aymanns, 2010), das Verlaufskurvenkonzept von Corbin und Strauss (2010), das von Goffman (1975) entwickelte Konzept der Stigmatisierung, die Dimension des Alters und Alterns (Pasero et al., 2007) sowie die Verwendung der Sprache als eine «verkörperte Sprache» (Krämer, 2001: 273) bildeten den Konzepthintergrund für die Leitfadenentwicklung. Eine offene Eingangsfrage sowohl in der Gruppendiskussion als auch in den Einzelinterviews danach, wie es sei, mit Demenz zu leben, machte die Dimension des «kritischen Lebensereignisse» und des Verlaufskurvenmodells sichtbar. Die Interviews hatten einen stark narrativ geprägten biografischen Fragehorizont. Die subjektiven Krankheitstheorien (z.B. im Kontext von Alternsprozessen), die Bedeutung der «kognitiven Einschränkung» für die Lebensqualität und deren konkrete Auswirkungen (z. B. in Bezug auf Stigmatisierung durch den sozialen Raum und den Verlust der Sprache) und die Bedeutung der Selbsthilfegruppe für die Bewältigung des Lebens mit Demenz bezeichneten zentrale Aspekte, die erfasst werden sollten. Die Gruppendiskussionen konzentrierten sich eher auf Aspekte des Erlebens der jeweiligen chronischen Erkrankung und der Funktion der Selbsthilfegruppe im Umgang mit dieser Erkrankung. Die Gruppendiskussionen und Interviews wurden jeweils auf einem Tonträger aufgezeichnet und vollständig nach anerkannten Transkriptionsregeln (in Anlehnung an Kuchartz, 2007) transkribiert. Zusätzlich wurden im Rahmen eines Forschungstagebuchs direkt nach den jeweiligen Gruppendiskussionen und Interviews Ablaufstrukturen, besondere Erlebnisse und weitere Gesprächsinhalte sowie Umgebungsaspekte festgehalten.

Die Auswertungsmethoden für die Einzelinterviews ebenso wie für die Aus- wertung der Gruppendiskussionen erfolgte in einem dreigliedrigen Verfahren nach den Vorgaben der rekonstruktiven hermeneutischen Textanalyse nach Panke-Kochinke (2004).

1. In einem ersten Schritt wurden alle transkribierten Texte einer immanenten Paraphrasierung unterzogen. Dabei wurden die von den Interviewpartner(inne)n beziehungsweise von den an der Gruppendiskussion Teilnehmenden Begrifflichkeiten weitgehend beibehalten (immanente Kategorienbildung).

2. Vor dem Hintergrund einer immanenten Kategorienbildung wurden in einem zweiten Schritt Themenfelder herausgearbeitet und für jedes Interview und jede Gruppendiskussion getrennt voneinander in einer zusammenfassenden Beschreibung festgehalten. Dieser Schritt der Auswertung ist vergleichbar mit dem von Bohnsack im Rahmen der dokumentarischen Methode als «formulierende Interpretation» bezeichneten Auswertungsschritt (Bohnsack, 2010).

3. In einem dritten Schritt wurden vor dem Hintergrund der ermittelten immanenten Kategorien in einem Strukturgitter (Greb, 2003; PankeKochinke, 2008) für jedes Interview beziehungsweise auch für jede Gruppendiskussion die zentralen Bezugsmuster respektive auch Widerspruchsfelder herausgearbeitet und die dazu gewählten immanenten Lösungsansätze festgehalten. Damit ließ sich die «Eigenlogik» des jeweiligen Textes erfassen.

Ergänzend wurde ein weiteres methodisches Verfahren eingesetzt. Die Verlaufsstruktur der Themenbereiche in den jeweiligen Texten wurde in einem grafischen Modell rekonstruiert. So wurde es möglich, die Schwerpunktsetzungen der jeweiligen Diskurse zu erfassen. Dieser weitere Auswertungsschritt war notwendig geworden, weil sich insbesondere die Interviews und die Gruppendiskussionen mit Menschen mit Demenz einer gewohnten Lesart teilweise entzogen.

Die immanent entwickelten Themenfelder, die Strukturgitter und die Verlaufsmuster dienten dann dazu, im Einzelfall ein zentrales Denkmodell in Bezug auf die gestellten Forschungsfragen $\mathrm{zu}$ erschließen. Diese wurden dann jeweils zunächst in den einzelnen Erhebungskategorien (Einzelinterviews und Gruppendiskussionen) miteinander verglichen. Auf diesem Wege ließ sich eine Form der reflektierenden Interpretation im Rahmen eines Fallvergleiches durchführen, die es erlaubte, über Gegenhorizonte Strukturmuster aus den Texten herauszuarbeiten. Nicht nur das, was augenscheinlich gesagt wurde, sondern auch der Kontext, der damit verbunden war, ließ sich so erschließen. Die Gemeinsamkeiten und Unterschiede der individuellen und Gruppenperspektive ließen sich so herausarbeiten.

Ein ethisches Clearing wurde durch die Ethikkommission der Deutschen Gesellschaft für Pflegewissenschaft (DGP) vor Beginn der Studie erteilt.

\section{Ergebnisse}

Aus den Interviews und den Gruppendiskussionen lassen sich in einem ersten Schritt mithilfe der rekonstruktiven hermeneutischen Textanalyse folgende immanent gewonnene Kategorien extrahieren, die einen Einblick geben in die Art und Weise, wie die Teilnehmerinnen und Teilnehmer von Selbsthilfegruppen sich selbst in ihrem Umgang mit der Demenz beschreiben. Diese Kategorien lassen sich zwar analytisch getrennt beschreiben, stehen aber im konkreten Lebenszusammenhang in einer inneren Verflechtung zueinander. Sie gehen dabei eine je individuelle Kombination ein. 
- Die Kategorien der Krankheitswahrnehmung, die Beschreibung des Krankheitsverlaufs und das subjektive Krankheits- und Gesundheitsbild definieren den Raum, in dem Demenz als Krankheit erlebt und gedeutet wird.

- Die Beschreibung des sozialen Nahraums (Partner(innen), Freunde/ Freundinnen und Bekannte, Mitglieder(innen) der Selbsthilfegruppe) lässt erkennen, welche Menschen und Menschengruppen wie und in welchem Umfang auf die Bewältigung der Demenz einwirken.

- Die biografischen Einflussfaktoren lassen sich vor allem in den Kategorien der Bedeutung der Ursprungsfamilie, begrenzt auch der Erwerbsarbeit fassen. Sie ermöglichen einen Zugriff auf unterstützende und behindernde Faktoren im Umgang mit der Erkrankung. Dieser Einflussfaktor wird subjektiv eher als weniger wichtig bezeichnet.

- Der zentrale innerpsychische Faktor der Suche nach einer «Gemeinsamkeit» macht deutlich, dass die sozialen Kontakte wichtig sind und wie und warum sie wichtig sind. Der Stellenwert der Selbsthilfegruppe im individuellen Lebenszusammenhang wird so deutlich erkennbar.

- Die Kategorie des «gelingenden Lebens» öffnet den Horizont für das Verstehen einer durchaus realistischen Einschätzung dessen, was wünschenswert und doch erreichbar erscheint.

- Die Kategorie der Mobilität beschreibt für Menschen mit Demenz nicht nur eine körperliche Bewegung im Raum, sondern auch eine intensive Auseinandersetzung mit dem inneren Erleben von Entwicklungsmöglichkeiten.

- Die Kategorie Medikamente erfasst aus der Innensicht der Betroffenen exemplarisch die unterstützende Funktion einer Intervention von außen und lässt erkennen, wo die Grenzen der innerpsychischen Beeinflussung liegen. Zwischen dem, was man selbst als etwas empfindet, was man dringend benötigt und dem, was man hinnimmt, erwächst ein individueller Handlungsspielraum.

In einem zweiten Schritt lässt sich vor dem Hintergrund der immanenten Kategorienbildung ein Handlungsmodell des Umgangs mit Demenz erfassen. Es wird als Modell der «inneren Sicherheit» (Abbildung 1) bezeichnet. Das Modell erfasst das Grundmuster des Aushandlungsprozesses, mit dem Menschen mit Demenz ihr Leben in der Beziehung mit anderen Menschen zwischen Kontrolle und Schutz so auszutarieren versuchen, dass es ihnen «guttut».

\section{Die immanenten Kategorien}

\section{Krankheitswahrnehmung}

Für Menschen mit Demenz ist die Demenz mit dem Begriff des «Vergessens» verbunden. «Vergessen» entsteht dann, wenn das Kurzzeitgedächtnis nicht mehr gut funktioniert. Das ist allerdings keine Krankheit, sondern ein
Problem, mit dem man einfach leben muss. Der «Kopf» vergisst etwas und als Sitz des Vergessens hat er wenig zu tun mit dem Menschen, der man war und auch bleibt. Die Krankheit ist das «Unbenennbare», das, was man nicht definieren kann. Sie bringt Unsicherheit und das Ziel ist es, Sicherheit zu gewinnen. Im «Hier und Jetzt» zu leben ist das Lösungsmuster. Depression ist demgegenüber beispielsweise eine Krankheit, die man mit Medikamenten behandeln kann. Sie ist ein Schleier, der das Denken verhindert und das Lebensgefühl negativ beeinflusst.

\section{Krankheitsverlauf}

Menschen mit Demenz beschreiben den Krankheitsverlauf vor dem Hintergrund ihres Lebensmusters des «Hier und Jetzt» als einen Zustand, der statisch ist. Die gefühlten Einordnungen bewegen sich in dem Verlaufsmuster zwischen «gut» und «nicht schlechter». Man ist «zufrieden».

\section{Krankheits- und Gesundheitsbild}

Demenz ist für die Betroffenen keine Krankheit. Während die Depression und das Vergessen spürbar und erlebbar sind, ist Demenz ein Begriff, der

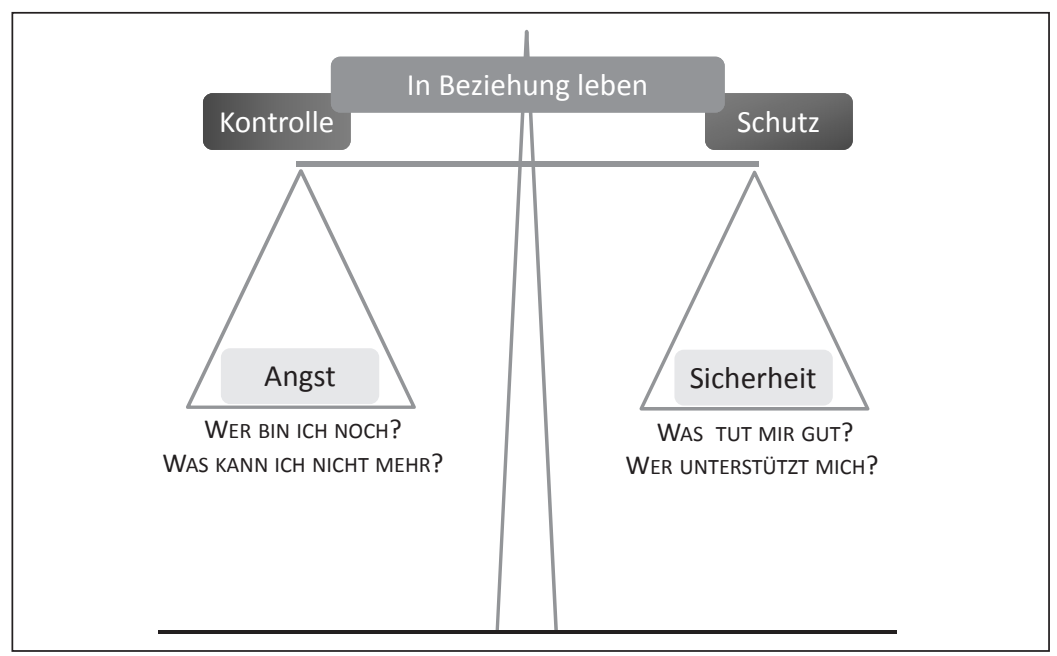

Abbildung 1: Das Modell der «inneren Sicherheit». 
wenig erkennbare Einschränkungen bedingt. Wenn man sich in der Position des «Hier und Jetzt» befindet und von einer Statik der Persönlichkeit ausgeht, dann ist sie kaum vorhanden. Man nimmt Medikamente, weil sie verordnet werden, man wird von Außenstehenden als dement bezeichnet, aber man empfindet sich in vielem einfach nur als einen alternden Menschen, der Probleme hat, die er bewältigen muss. Das «Unbenennbare» ist ein Rekurs auf diese Unsichtbarkeit einer Erkrankung, die als Krankheit nur wahrgenommen wird, weil sie von außen stigmatisierend als Begriff auf die eigene Person angewendet wird.

\section{Partner(innen)}

Wenn sie vorhanden sind, haben sie eine wichtige Funktion und Rolle im Umgang der Betroffenen mit ihrer Erkrankung. Erkennbar werden Unterschiede in der Funktion, die sie aus der Sicht der jeweils von der Erkrankung betroffenen Menschen, einnehmen. Wesentliche Unterscheidungsfaktoren sind das Alter und das Geschlecht. Menschen mit Demenz, die weiblich sind, weisen ihrem Partner eine entscheidende Rolle im Umgang mit der Krankheit zu. Sie sollen den Sicherheits- und Schutzraum garantieren, den sie benötigen, um das «Hier und Jetzt» gut leben zu können. In dem Bemühen um positiv bestimmte Selbstregulierung und Aktivierung ihrer Ressourcen fühlen sie sich dann gestört, wenn ihr tägliches Gegenüber sie auf eine für sie unangenehme und verletzende Art und Weise darauf hinweist, dass sie vergesslich und krank sind. In diesem Moment werden sie in ihrem inneren Schutzraum verunsichert. Das tut ihnen nach ihren eigenen Aussagen nicht gut. Die Reaktionsformen sind individuell verschieden: man kann das Problem argumentativ nach außen verlagern («Männer sind eben anders als Frauen»), man kann neue
Kommunikations- und Konfliktlösungsstrategien einsetzen oder man kann auch einfach versuchen, den eigenen Raum der Selbstentwicklung zu vergrößern. Trotzdem bleiben Partner(innen) ein Problem, weil sie intensiv und nahe und zugleich mit einem hohen Verletzungspotenzial ausgestattet sind. Frauen als Partnerinnen können Schutzfunktionen aus der Sicht des betroffenen Mannes mit Demenz übernehmen, die ein Abgebenkönnen von Verantwortung bedeuten. Das wird einfach so angenommen und reduziert das Wohlbefinden nicht. Auch dann - aber das ist eine Vermutung - wenn diese Partnerin sich über das Leben mit dem Menschen mit Demenz beklagen, ist das nicht unbedingt eine Störung für den Betroffenen, solange dieser Schutz bestehen bleibt. Sind die Partnerinnen jünger, finden auch aufgrund des Faktors Erwerbsarbeit die Aushandlungsprozesse in einem anderen Raum statt. Das ist allerdings in dem Material nur ansatzweise zu erkennen.

\section{Die "Ursprungsfamilie»}

Die Auseinandersetzung mit der Frage, was einen in seinem Verhalten geprägt hat und wie man aktuell damit umgehen kann, berührt den Kern einer Auseinandersetzung mit der «Ursprungsfamilie». Ausgelöst auch durch therapeutische Interventionen aber auch einfach durch die Erzählung der Biografie im Rahmen der Interviews, wird erkennbar, welche Bedeutung der «Ursprungsfamilie» aus der Sicht der Menschen selbst zukommt. Es ist dabei nicht zentral, dass etwas erinnert wird, es stellt sich eher die Frage, welcher Stellenwert dem Erinnerten in Bezug auf die Bearbeitung der Gegenwart zugemessen wird.

Menschen mit Demenz mit ihrem Modell des im «Hier und Jetzt» leben, sind weniger bereit und bestrebt, von ihrer Vergangenheit zu erzählen. Wenn sie das im Gespräch tun, dann eher im
Hinblick auf kurze Sequenzen von Erfahrungen, die erklären sollen, warum beispielsweise Klavierspielen etwas mit dem Wiederaufnehmen von Bekanntem zu tun hat. Der Bezug zur «Ursprungsfamilie» erscheint indifferent. Geschwister und Eltern sowie Großeltern sind ein Teil der Vergangenheit und sie sind im «Hier und Jetzt» meistens nicht präsent. Die Geschwister sind so präsent, wie sie auch da sind und der Rest der Familie ist im Rahmen von Familienfeiern einfach vorhanden.

\section{Freunde/Freundinnen und Bekannte}

Freunde/Freundinnen und Bekannte sind wichtig. Sie sind nicht Familie. Sie decken bestimmte darüber hinausgehende Bedürfnisstrukturen ab. Sie dienen dazu, das Selbstkonzept zu entwickeln. Für die interviewten Menschen mit Demenz ist der Grad der Wichtigkeit von Freunden/Freundinnen und Bekannten unterschiedlich hoch und wird unterschiedlich gelebt. Von der ehrenamtlichen Tätigkeit und dem Engagement in der Selbsthilfegruppe über gemeinsame Urlaube mit anderen sowie gemeinsame Sport- und Freizeitveranstaltungen reichen die je individuell als hinreichend ober befriedigend wahrgenommen Kontakte. Sie sind «wichtig» bis «ganz wichtig». Der Begriff der «Gemeinsamkeit» berührt dabei erneut das Gefühl des «Guttuns». Menschen, die als nicht guttuend empfunden werden, werden aussortiert. Stigmatisierungsprozesse im Privatraum werden so zumindest minimiert.

\section{Erwerbsarbeit}

Für den größten Teil der Menschen mit Demenz bedeutet die Diagnose in Bezug auf die Erwerbsarbeit konkret wenig: Sie leben bereits in einem Zustand der Rente. Für wenige andere bedeutet diese Diagnose eindeutig das Ende der Erwerbstätigkeit. Dann allerdings entsteht ein Bruch, mit dem man umzugehen lernen muss. Erwerbsarbeit in 
einer eher biografisch historischen Rückschau hat unter der Perspektive des Hier und Jetzt eine Bedeutung dann, wenn daraus aktuelle Handlungsmuster abgeleitet werden können. Yogalehrerin gewesen zu sein und aktuell die Verbindung zwischen Körper, Seele und Geist anzustreben bedingen sich so. Gruppenprozesse wichtig zu finden, wenn man selbst analytisch gearbeitet hat, stellt eine ebensolche Verknüpfung her. Auch die Blickrichtung auf die eigene Erkrankung wird beispielsweise von einem ehemaligen Mediziner oder einer ehemaligen Medizinerin anders betrachtet. Erwerbsarbeit bleibt also als Erfahrung und Erinnerung eine zentrale Quelle der Identitätsbildung, die sich dann auch in dem Umgang mit der Demenz, der Suche nach Bewältigungsstrategien (z.B. ein therapeutischer, körperbezogener, psychiatrischer Zugriff) und den jeweiligen subjektiven Krankheitsund Körperfunktionsbildern widerspiegelt.

\section{Gemeinsamkeit}

Der Begriff der Gemeinsamkeit schreibt für Menschen mit Demenz ein Gefühl, ein Bedürfnis und eine Erfahrung. Sie ist eine zentrale Variable, wenn erklärt werden soll, was die Selbsthilfegruppe, Freunde/Freundinnen, Bekannte, die Angehörigen und andere Gruppenzusammenhänge bedeuten. Gemeinsamkeit ist die Bezeichnung für etwas Wünschenswertes, weil sie einen zentralen Faktor auf dem Weg zu einem guten Leben darstellt. Gemeinsamkeit hat etwas zu tun mit Erfahrungen der Sicherheit und Unterstützung, ist aber mehr als das, nämlich auch ein Gefühl der Freude, die über eine alltägliche Gemeinschaft hinausgeht. Gemeinsamkeit kann gelebt und erlebt werden über unterschiedliche Personen und Gruppen: den/die Partner(in) durch den Wunsch und das Bedürfnis nach einer tiefen Verständigung, die Selbst- hilfegruppe durch das gemeinsame Sprechen und Verstehen, Freunde/ Freundinnen und Bekannte in dem gelebten partiellen Beisammensein mit andern im Urlaub oder in der Sportgruppe. Gemeinsamkeit entsteht hier in der Ermöglichung von echtem Austausch. Gemeinsamkeit bezeichnet etwas, das dann, wenn es passiert, sehr selbstverständlich wirkt und dann, wenn es fehlt, als Entzug von etwas Wesentlichem empfunden wird. Gemeinsam mit sich selbst kann man nicht sein, man kann aber wohl mit anderen gemeinsam etwas Wesentliches für sich selbst umsetzen und lernen. In diesem Sinne ist die Selbsthilfegruppe für Menschen mit Demenz etwas Gemeinsames dann, wenn Ansätze eines solchen Erlebens möglich sind.

\section{Gelingendes Leben}

Das gelingende Leben ist ein Leben, das gut ist. Gut ist zwar eine statische Beschreibung eines Zustands und gelingend ein Prozess, sie hängen aber an einem bestimmten Punkt zusammen Gelingend meint für Menschen mit Demenz dabei, dass das, was als gut bezeichnet werden kann, immer wieder so austariert werden muss, dass es diesem Zustand nahekommt. Gelingend ist somit eine Prozesskategorie, die zugleich ein hohes Justierungspotenzial ausweist. Die Faktoren, die zu einem gelingenden Leben beitragen können, werden in der Selbsthilfegruppe erzählt. Sie bilden eine wesentliche Grundlage der Gemeinsamkeit. Die Botschaft lautet: erkläre du mir, wie du dein Leben gut führst und ich erkläre dir, wie ich das mache. So können wir voneinander lernen und erzeugen in dem Akt der Mitteilung bereits die Voraussetzung für ein gelingendes Leben Das ist die Grundlage, die auch für Selbsthilfegruppen ausgewiesen wird. Das gelingende Leben manifestiert sich in einer Art Heldenmythos, durch den man sich selbst in dem Akt des Er- zählens und in der Vermittlung von Geschichten stärkt. Man lernt aneinander und miteinander, wie man mit dem Leben umgehen kann.

Wenn es um die Reflexion des eigenen Lebens geht, das nicht in der Gemeinschaft der Selbsthilfegruppe stattfindet, dann werden die Geschichten von einem gelingenden Leben mit Geschichten konfrontiert, die von Problemen und Schwierigkeiten berichten. Da Bewältigung auf ein Weiterleben mit der Erkrankung zielt und zielen muss, werden Justierungen erkennbar, wie man zumindest ein Gleichgewicht zwischen dem herstellen kann, was man für gut hält und dem, was man akzeptieren muss.

\section{Mobilität}

Mobilität heißt Bewegung oder Beweglichkeit. Worauf sich jemand zubewegt oder was er benötigt, um beweglich sein $\mathrm{zu}$ können, ist unterschiedlich definiert.

Für Menschen mit Demenz bedeutet sie nicht nur Bewegung im äußeren Raum, sondern vor allem auch Bewegung entsprechend einem Aktivitätsmodell im inneren Raum mit sich selbst. Konzepte des Zusammenwirkens von Körper und Geist werden zu einer argumentativen Klammer, warum man Sport machen sollte, warum es wichtig ist, sein Gedächtnis zu schulen oder mit Freunden/Freundinnen zu kommunizieren. Wenn das Gedächtnis nachlässt und das Vergessen spürbar wird, ist es wichtig, mobil zu bleiben, um den inneren Verfall aufhalten zu können. Die Frage der äußeren Bewegung als Bewegung im Raum und der Grad der inneren Bewegung durch Aktivität kennzeichnet im Umgang mit der Demenz Entwicklungsprozesse. Das Neue erfahren und das Bekannte ausbauen zu wollen, sind solche Justierungsprozesse im Verlauf einer Erkrankung, die sich erkennen lassen. 


\begin{abstract}
Medikamente
Medikamente haben eine Funktion und eine Aufgabe. Sie helfen, das Leben mit einer chronischen Erkrankung zu leben und ermöglichen es, einen Schnittpunkt zum System der Medizin zu definieren. Ein Zustand, der den Einsatz von Medikamenten befürwortet, ist die Depression. Depressionen verhindern aus der Sicht der Betroffenen die Auseinandersetzung mit der chronischen Erkrankung. Sie verschleiern das Gemüt und führen zu einer Selbstaufgabe. Gut mit der Krankheit umgehen zu können, heißt auch immer, dass man sich seelisch dazu in der Lage sieht. Dazu gehören neben therapeutischen Interventionen eben auch Medikamente. Menschen mit Demenz stehen den Medikamenten gegen Demenz indifferent gegenüber. Sie wissen nicht genau, ob sie wirken und wie sie wirken, aber sie nehmen sie. Sie helfen gegen die Vergesslichkeit - das ist die Position dazu.
\end{abstract}

\section{Das Modell der «inneren Sicherheit»}

Vor dem Hintergrund der immanenten Kategorienbildung lässt sich in einem weiteren Schritt ein Handlungsmodell der Bewältigung als ein Modell der Suche nach «innerer Sicherheit» (Abbildung 1) erschließen, das genutzt werden kann, um den je individuellen Fall in seinen Bezugsmustern einordnen zu können. Es spiegelt also in diesem Stadium der Analyse nicht mehr die individuelle Ebene selbst wider, sondern erfasst die Grundmuster respektive die Bestimmungsvariablen der Bewältigung im Umgang mit der Demenz. Folgender Interpretationsrahmen lässt sich aufspannen.

Auf der einen Seite geht es Menschen mit Demenz, die sich in einer Selbsthilfegruppe engagieren, nach ihren eigenen Aussagen darum, das Leben gelingen zu lassen. Die Demenz als
Krankheit wird so in den eigenen Lebenszusammenhang integriert, dass es einem «gut» geht. Ein gelingendes Leben zu führen, heißt für sie auch, ein Leben ohne Klagen führen zu können. Es ist gekennzeichnet durch das Bemühen um konkrete Lösungsstrategien, wenn Probleme auftreten.

Menschen mit Demenz im Frühstadium arbeiten so an einem Modell der «inneren Sicherheit», das es ihnen erlaubt, in Beziehungen zu leben, das heißt die Gemeinsamkeit mit anderen als Bereicherung und Unterstützung zu erfahren und sich dabei soweit wie möglich selbst entwickeln zu können. Sie wollen für sich selbst herausfinden, wie sie für sich selbst am besten sorgen können. Zwischen einer Kontrolle von außen, die ihnen Angst macht und einem Bedürfnis nach Schutz, das ihnen eine sichere Grundlage für ihr Leben gibt, setzen sie sich mit der Abhängigkeit auseinander, die sie dadurch erleben, dass sie in Beziehungen mit anderen Menschen leben und auch leben wollen. Die Suche nach dem, was einem guttut und dem Grad von Unterstützung, die man benötigt, um sich sicher zu fühlen, muss vor dem Hintergrund einer existenziell bedrohlichen Frage nach der eigenen Identität (Wer bin ich?) und der Einschränkung der Kompetenzen (Was kann ich nicht mehr?) beantwortet werden. Sie streben nach einer Art der Aktivität, die an etwas Bekanntes anknüpfen kann und in dem Muster der Beziehungen, die ihnen guttun das Neue im bereits bekannten zu entdecken erlaubt.

Erkennbar wird so, wie Menschen mit Demenz nach dem suchen, was sie als gut tun bezeichnen. Das ist dann jeweils unterschiedlich, aber in der Wirkung gleich: nur das tut gut, was Sicherheit gibt und diese lässt sich nur dann erreichen, wenn man geschützt wird und sich auch schützen lässt. Sicherheit steht der Angst entgegen. Menschen mit Demenz im Frühstadium haben dabei mit dem besonders starken Problem der Entmündigung zu kämpfen, die sie, obwohl sie sich zunächst auch gar nicht besonders behindert fühlen, ertragen müssen. Ein inneres Gleichgewicht zu finden, das Selbstkonzept anzupassen und eine Form der Akzeptanz durch andere zu finden, ist für sie eher im Vordergrund.

Menschen mit Demenz sind dabei in einem hohen Maße von den Beziehungen zu anderen Menschen abhängig, die in ihrem näheren Umfeld leben. Insbesondere den Partnern kommt dabei eine hohe Relevanz zu. Der Begriff der «Kontrolle» ist für Menschen mit Demenz negativ besetzt, insofern sie in einigen Segmenten der Kontrolle Entmündigung erleben. Sie sehen sich bisweilen gezwungen, Dinge aufzugeben, die sie eigentlich nicht aufgeben wollen. Kontrolle steht dem Wunsch nach einer Unterstützung und Hilfe entgegen, die sich daran orientiert, was man braucht und haben möchte.

\section{Zusammenfassung}

Menschen mit Demenz beschreiben ihren Umgang mit der Erkrankung als ein beständiges Austarieren zwischen dem Wunsch nach und dem Erleben eines «gelingenden Leben» und der Erfahrung von Entmündigungsprozessen. Auf der Seite des «gelingenden Lebens» wird für sie Selbstwirksamkeit und Unterstützung von außen so austarierbar, dass es ihnen «gut» geht oder sie zumindest «zufrieden» sind. «Gemeinsamkeit» mit anderen Menschen ist für die Umsetzung eines solchen «gelingenden Lebens» zentral. Der Begriff bezeichnet eine Vielfalt von Möglichkeiten, mit anderen Menschen in sozialen Bezügen zu leben: von der Partnerschaft über die «Ursprungsfamilie», Freunde/Freundinnen, Bekannte und die Selbsthilfegruppe. «Gemeinsamkeit» beschreibt für sie das 
Gefühl einer echten und tiefen situativen Verbundenheit mit anderen Menschen. In diesem Begriff der «Gemeinsamkeit» wird auch der Stellenwert der Selbsthilfegruppe deutlich. In der Gruppe realisiert sich für sie ein wesentliches Element der «Gemeinsamkeit». Es ist nicht nur wichtig, mit anderen Menschen an einem Ort $\mathrm{zu}$ sein, sondern sich auch von ihnen verstanden, akzeptiert und damit geachtet zu fühlen. Die Selbsthilfegruppe ermöglicht so aus der Sicht der Menschen mit Demenz eine Gemeinsamkeit, die einen Austausch über Probleme, aber vor allem auch Faktoren des gelingenden Lebens und darüber hinaus eine als produktiv bezeichnete Auseinanderersetzung mit sich und der Krankheit möglich macht. Man wird auf eigene Probleme und Unstimmigkeiten hingewiesen, ohne dass man direktiv gezwungen wird, die Ratschläge auch umzusetzen. Die «innere Sicherheit» kann sich in diesem erweiterten Raum der Information, der Sympathie und des vergleichsweise hohen Grads einer praktizierten Anerkennung gestärkt werden.

Menschen mit Demenz beschreiben für sich aber auch die Gefahr, dass in dieser «Gemeinsamkeit» die Angriffsfläche für Entmündigungsprozesse gegeben ist. Auf dieser anderen Seite der Erfahrung müssen sie sich damit auseinandersetzen, dass die Menschen, denen sie im familiären und räumlichen Rahmen nahe sind - im gegebenen Fall sind das die Lebenspartner(innen) zugleich eine schützende und verletzende Funktion haben. Die Selbstwirksamkeit kann empfindlich gestört werden und kann auch kaum durch eine Intervention von außen aufgelöst werden. Wenn es zu Entmündigungsprozessen kommt, dann finden diese in diesem engsten sozialen Raum statt, also genau in dem Bereich, in dem die «Gemeinsamkeit» angestrebt und auch als Schutz und Sicherheit gebraucht wird. Die Systeme der «Inneren Sicherheit» werden so in ihrem Kern angegriffen. Und genau an diesem Punkt wird die Funktion einer Selbsthilfegruppe erneut sichtbar: sie bietet einen unterstützenden Raum, in dem genau diese Probleme angesprochen und Lösungsmuster entwickelt werden können.

\section{Diskussion}

Einleitend war die Frage gestellt worden, wie sich Menschen mit Demenz im Frühstadium ihrer Erkrankung selbst wahrnehmen und welchen Einfluss die Intervention einer Selbsthilfegruppe auf ihr Selbstkonzept hat. Die immanent gewonnenen Kategorien und das Modell der «inneren Sicherheit» geben aus der Sicht der interviewten Menschen mit Demenz erste Antworten. Wie binden sich diese Erkenntnisse in den wissenschaftlichen Diskurs ein? Der Fokus liegt im Folgenden darauf, auf den möglichen Mehrwert der gewonnenen Ergebnisse zu verweisen.

Ein gelingendes Leben ist aus der Sicht der interviewten Menschen mit Demenz ein Leben, in dem es gelingt, in einem Ausloten zwischen Kontrolle und Sicherheit mit anderen Menschen in Beziehung zu bleiben. Das bedeutet zudem, dass Unterstützung in dem Maße angefragt werden kann und auch erteilt wird, wie sie für den Menschen selbst als notwendig und wünschenswert bezeichnet wird und nicht eine Hilfe aufoktroyiert wird, für die gar kein Bedarf besteht. Damit wäre aus der subjektiven Sicht ein wesentlicher Bestandteil von Lebensqualität beschrieben, wie sie sich auch im wissenschaftlichen Diskurs wiederfindet. So ist nicht nur für Holzhausen (Holzhausen et al., 2009) der Begriff der Lebensqualität ein Schlüsselindikator, um zu erfassen, wie man das Wohlbefinden und die Funktionsfähigkeit von Menschen mit Demenz im Umgang mit Symptomen in der Verbindung zwischen Innen und Außen so fördern kann, dass sie adäquat damit umgehen können. Dieses ist für ihn mit dem Behandlungsziel des «guten Lebens» im Alter verknüpft (Holzhausen et al., 2009: 335), die man dem «gelingenden» Leben an die Seite stellen kann. Wenn Holzhausen Lebensqualität definiert, dann tut er das im Prinzip, indem er den Begriff des Bedürfnisses und der Bedarfe zusammenbringt. Der Begriff «adäquat» verweist auf den Vermittlungsaspekt zwischen Innen und Außen, der nicht genau definiert wird. An dieser Stelle der Vermittlung sitzt, so ein Ergebnis der Studie SEIN, der Begriff der «inneren Sicherheit» und zwar aus der Sicht von Menschen mit Demenz in einer doppelten Konnotation: als Bestimmung des subjektiven Wohlbefindens im Erleben eines «Guttuns» und im Fordern und Annehmen von Hilfsangeboten von außen. Der Begriff des Selbstkonzepts bezeichnet die Stelle, an der die Umwelt und das individuelle Erleben zusammenkommen und sich als förderlich oder behindernd erweisen.

Als zentrales Problem ließ sich in dieser Untersuchungsgruppe das Erleben eines Entmündigungsprozesses erfassen, der insbesondere auch über Partnerinnen und Partner transportiert werden kann. Entmündigung wurde als eine spezifische Form der Stigmatisierung erkennbar, die durch den Zwang zu einem scheinbar freiwilligen Verzicht in Erscheinung tritt. Diese Erkenntnis ergänzt kritisch - aus der Perspektive der Menschen mit Demenz - Forschungen, die die Rolle und Funktion der Angehörigen in der Bewältigung der Demenz zum Inhalt haben (Langehenning \& Obermann, 2006).

Das «Vergessen» konnte für die interviewten Menschen mit Demenz als das zentrale Problem der Demenz geortet 
werden. «Vergessen» entsteht aus ihrer Sicht dann, wenn das Kurzzeitgedächtnis nicht mehr gut funktioniert. Die dadurch entstehenden Probleme in der Alltagsbewältigung sind dann für sie weitgehend lösbar, wenn sie die Unterstützung ihres sozialen Umfelds dabei bekommen. «Vergessen» ist in ihrer Sprache zwar die Bezeichnung für eine erlebte Einschränkung, die aber vermutlich eine andere Konnotation hat als der Begriff des «Gedächtnisverlustes» (Hauser, 2009: 80) oder die als Leitsymptom der Demenz bezeichnete «kognitiven Störung». In der Auseinandersetzung mit dem «Vergessen» entwickeln sie Strategien im Umgang damit, die sie für sich als angemessen bezeichnen. In ihrer eigenen Wahrnehmung praktizieren sie so bereits einen Schritt aus der Defizitorientierung heraus, der sich in der wissenschaftlichen Diskussion erst andeutet.

Interessanterweise spielt die Frage der Diagnosestellung und Phaseneinteilung im Umgang mit der Erkrankung kaum eine Rolle. Die interviewten Menschen mit Demenz ordnen sich nicht in eine bestimmte Phase der Demenz ein. Sie beschreiben lediglich die an ihnen durchgeführten Untersuchungen, kritisieren die Form der Tests und sprechen über ihre Medikamenteneinstellung. Dass sie etwas vergessen, wissen sie. Dass man sie danach fragt, was sie eigentlich nicht wissen können, halten sie für wenig produktiv. Trotz der medizinischen Diagnosestellung ist für sie mit dem ihnen zugeteilten Begriff der Demenz nicht unbedingt ein Krankheitsempfinden verbunden. Dieses Verhalten ließe sich zumindest in der Deutung dieses Begriffs, wie sie auch Stechl vertritt (Stechl, 2006a; Stechl et al., 2006b; Stechl et al., 2007), als eine Form der durchaus positiven Bewältigungsstrategie im Sinne einer fehlenden Krankheitseinsicht (Anosognosie) bezeichnen. Ob die Frage der fehlenden
Krankheitseinsicht nicht auch als durchaus berechtigter Zweifel an den diagnostischen Möglichkeiten des medizinischen Fachpersonals interpretiert werden kann, wäre zu überlegen. Die im Rahmen der Studie interviewten Menschen mit Demenz registrieren zwar depressive Verstimmungen. Sie stellen von sich aus aber keine Verbindung zu den «Leerstellen» in der Erinnerung her (Baer, 2007: 80). Angst und Unsicherheit werden zwar benannt, aber nicht unbedingt mit Depression gleichgesetzt. Angst wird auch gedeutet als durchaus reale Folgeerscheinung einer Intervention von Seiten des Angehörigen oder zum Beispiel die tägliche Kontrolle durch professionell Pflegende. Aus der individuellen Sicht gibt es für die Depressionen ein probates Mittel: die Medikamenteneinnahme. Sie eröffnet die Möglichkeit, sich wieder in sozialen Bezügen zu erleben. Den Medikamenten, die sie gegen Demenz verabreicht bekommen, weisen sie zwar nicht unbedingt eine Wirksamkeit in Bezug auf das «Vergessen» zu, lehnen sie aber grundsätzlich auch nicht ab. Selbsthilfegruppen werden generell in der Literatur als hilfreich in der Unterstützung von und für Menschen mit Demenz eingeschätzt. Dem ist grundsätzlich aus der Sicht der an der Studie beteiligten Interviewpartner(innen) zuzustimmen. Die Analyse lässt auch genauer erkennen, worin die Unterstützungsfunktion gesehen wird: in der Ermöglichung eines Gefühls von «Gemeinsamkeit» auch außerhalb der Angehörigen und Freunde/Freundinnen. Zentral ist der Aspekt der Akzeptanz und gegenseitigen Anerkennung. Dass dabei eher die gelingenden Geschichten einer Lösung von Problemen in den Vordergrund gerückt werden, erscheint im Sinne einer Gesundheitsförderung nicht unbedingt schädlich und verweist auf einen eher salutogenetischen Blick. Es ist zu vermuten, dass sich in den Selbsthilfegruppen für Menschen mit Demenz also die Menschen engagieren, die ein solches Potenzial bereits mitbringen. Kritisch wäre allerdings anzumerken, dass die Selbsthilfegruppen im Sinne des Stigmatisierungskonzepts von Goffmann (1975) auch eine gut funktionierende Form der internen Abgrenzung praktizieren und damit zumindest im gesellschaftlichen Feld der Wahrnehmung weniger die Stigmatisierung beziehungsweise Entmündigung selbst, die ja als von außen kommend empfunden wird, begrenzen oder gar reduzieren ${ }^{4}$.

Die Ergebnisse der Studie liefern zwar einen Hinweis darauf, wo Ressourcenpotenziale im Umgang mit Entmündigungsprozessen liegen können. Inwieweit allerdings die Formen der Entmündigung, die subjektiv von den Betroffenen erlebt werden, überhaupt ressourcenorientiert gelöst werden können, muss zumindest angezweifelt werden. Entmündigung ist ein Aberkennungsprozess von menschlichen Grundrechten. Er stellt das negative Pendent zu Selbstbestimmungsprozessen dar und trifft damit den Kern eines diskriminierenden Umgangs mit Menschen mit Demenz. Wenn eingangs die Vermutung aufgestellt wurde, dass sich in der Literatur bereits Hinweise darauf finden, dass das defizitorientierte Bild eines Menschen mit Demenz langsam an Boden verliert und eine gesellschaftliche Integration zumindest ansatzweise zu finden ist, dann weisen die Ergebnisse der Studie möglicherweise eher darauf hin, dass diese programmatisch vorgetragenen Perspektiven mehr Wunsch als Realität sein könnten.

Des Weiteren ist festzuhalten, dass ein Faktorenbündel, das für die Entwick-

\footnotetext{
4 Der Begriff der Stigmatisierung wird nach Goffman (1975: 11) «in Bezug auf eine Eigenschaft gebraucht, die zutiefst diskreditierend ist.» Jemand ist in unerwünschter Weise anders, als man es antizipiert hatte (Goffmann, 1975: 13).
} 
lung einer Passgenauigkeit von Interventionen zentral ist, bisher nicht erschlossen werden konnte. Passgenau sind, so die zu begründende Vermutung, nur die Interventionskombinationen, die auch berücksichtigen, wie sich im Laufe der Erkrankung die Bedarfe und Bedürfnisse der Betroffenen ändern können. Es fehlt also bisher noch ein Studiensegment, das die Langzeitperspektive, die die Verlaufskurvenmodelle der Ein- und Anpassung respektive der Probleme mit derselben rekonstruiert. Die Frage ist bisher noch keineswegs beantwortet, wie man die Dynamik eines solchen Bewältigungsprozesses individuell rekonstruieren und im Vergleich spezifische Muster für ein bestimmtes Krankheitsbild und einen bestimmten Verlauf für eine Person erfassen kann. Die Typisierung solcher individuellen Verlaufsmuster könnte dann dazu dienen, um als Instrument nicht neue Interventionen $\mathrm{zu}$ «erfinden», sondern $\mathrm{zu}$ erfassen, welche Kombination von welchen Interventionsschritten ein Mensch mit Demenz zu welchem Zeitpunkt benötigt. Auch an diesem Punkt sind noch keine eindeutigen Antworten zu geben. Erkennbar im Rahmen der Studie ist aber bereits das, was Menschen mit Demenz im Frühstadium ihrer Erkrankung keinesfalls brauchen und was für sie hilfreich ist. In der Langzeitperspektive ließen sich möglicherweise weitere wichtige Faktoren im Krankheitsverlauf herausarbeiten. Im Sinne von Corbin und Straus (2010), vor dem Hintergrund von Schütze (1981) und im Rahmen der Lebenslaufforschung wird es entsprechend darum gehen müssen, diese Langzeitperspektive durch Kohortenstudien zu entwickeln.

\section{Schlussfolgerungen}

Vor dem Hintergrund der Forschungsergebnisse lassen sich folgende zentrale Schlussfolgerungen für den Um- gang mit Menschen mit Demenz im Frühstadium ihrer Erkrankung, die sich in Selbsthilfegruppen engagieren, für die Praxis ableiten.

Wenn Menschen mit Demenz im Frühstadium ihrer Erkrankung sich darüber äußern, wie es für sie ist, mit Demenz zu leben, steht für sie auf der negativen Seite das Problem der Entmündigung im Vordergrund. Entmündigung tritt für sie insbesondere dann ein, wenn sie ungewollt Interventionen ertragen müssen oder Dinge und Gewohnheiten aufgeben müssen, obwohl sie sich persönlich noch keineswegs als untüchtig begreifen. Fürsorglich ist für sie demgegenüber ein Verhalten, dass sie als Schutz deshalb empfinden, weil sie im Falle eines krankheitsbedingten Versagens auf Anfrage eine passende Unterstützung erhalten. Es kann sich dabei um Angehörige, aber auch andere Menschen in ihrem sozialen Umfeld handeln, die ihnen hilfreich zur Seite stehen. In Bezug auf Beratungs- und Unterstützungsangebote für Menschen mit Demenz in einer frühen Phase ihrer Erkrankung wäre also darauf zu achten, dieses labile System zwischen Hilfsangeboten und Fürsorge angemessen zu reflektieren. Für professionell Pflegende wäre besonders wichtig, dass sie eine selbstreflexive Haltung der Geduld und Akzeptanz Menschen mit Demenz im Frühstadium der Erkrankung gegenüber gewinnen. Die Unterstützung und Begleitung von nahen Angehörigen von Menschen mit Demenz im Frühstadium der Erkrankung, insbesondere der Lebenspartner(in) bestätigt sich vor dem Hintergrund der Ergebnisse der Studie als sinnvoll.

In den Selbsthilfegruppen, die als Support Groups durchgeführt werden, wird es dementsprechend von den Menschen mit Demenz als wohltuend und außerordentlich hilfreich erlebt, wenn man einfach beschreiben kann, wie es einem geht, ohne dass daraufhin
Ratschläge für ein richtiges oder falsches Verhalten erfolgen. Die Verständigung untereinander, für einige der Beteiligten aber auch die Sicherheit, eine Unterstützung von außen zu haben, bestimmt die Erfahrung in einer solchen Gruppe. Selbsthilfegruppen unterstützen so Prozesse der sozialen Integration. Sie ermöglichen darüber hinaus auch eine Form der Beratung und Selbsthilfe, die Akzeptanz findet. Dieses Modell der Hilfe zur Selbsthilfe als Unterstützungsform ließe sich als konzeptioneller Ansatz noch stärker als bisher geschehen auf andere Angebotsformen wie das Gedächtnistraining übertragen.

An analysis of the individual perception and coping strategies of people with dementia in the early stage of their disease in accordance to the function and effectiveness of self-help groups on the basis of self-expression Society paints an inconsistent picture of people with dementia: on the one hand they are people who can basically think and act (even) self-determinate. But on the other hand they need support and help from outside in daily routine. Self-help groups are a possible form of support. There are actually no research results to decide if this form of intervention is appropriate to people with dementia or not.

The question must be answered in which way people in the early stage of dementia perceive their illness and what kind of influence the intervention of a support group has on their self-concept. In the first survey period five focus groups and eight narrative biographically oriented interviews are performed. The following results are noted: People with dementia are ex- 
posed to processes of incapacitation. It is well intentioned (in order to help them), but in reality they are forced to do something they probably don't like. They must bear unwanted interventions. In contrast, the self-help groups promote recognition processes. Finding internal security and being able to accept the illness are some of the most important functions. Independent living with dementia is supported. Two conclusions can be drawn:

Forms of incapacitation from outside must be reduced. Self-help groups in a support role should accordingly supply the existing care services for people with dementia. The support of selfhelp groups should be the provision/ improvement of the existing care services for people with dementia.

Key words: people with dementia, selfhelp group, intervention

\section{Literatur}

Altinisik, S. (2009). Bewältigungsmechanismen von Demenzerkrankten im Frühstadium. Eine qualitative Interviewstudie mit Teilnehmern einer Selbsthilfegruppe der Alzheimer Gesellschaft e. V. Saarbrücken: VDM.

Baer, U. (2007). Innenwelten der Demenz. Das SMEI-Konzept. Eine leibphänomenologische Untersuchung des Erlebens demenzkranker Menschen und der Entwurf einer Begleitung, die ihr Erleben würdigt, Neukirchen-Vlyn.

Bell, V.; Troxel, D. (2006). A Word from the Wise: What Persons with Early Dementia Tell Us about Activities. In: Demenz - eine Herausforderung für das 21. Jahrhundert. 100 Jahre Alzheimer-Krankheit (Tagungsreihe der Deutschen Alzheimer Gesellschaft e. V., Bd. 6), 139 - 148.

Corbin, J. M.; Strauss, A. L. (2010). Weiterleben lernen. Verlauf und Bewältigung chronischer Krankheit, Bern: Hans Huber.

De Boer, M. E.; Hertog, C. M.; Dröes, R. M.; Riphagen, I. I.; Jonker, C.; Eefsting, J. A. (2007). Suffering from dementia - the patient's perspective: a review of the literature. In: International Psychogeriatrics,19 (6), $1021-1039$.

Bohnsack, R. (2010). Rekonstruktive Sozialforschung. Einführung in qualitative Methoden. Opladen: Barbara Budrich.

Breuer, F. (2009). Reflexive Grounded Theory. Eine Einführung für die Forschungspraxis. Wiesbaden: VSVerlag.

Demenz Support Stuttgart (Hrsg.) (2009). Hearing the Voice Revisited. Teil 1: Einbindung und Teilhabe. Stuttgart: Demenz Support Stuttgart.

Demenz Support Stuttgart (Hrsg.) (2010a). «Ich spreche für mich selbst». Menschen mit Demenz melden sich zur Wort. Frankfurt/ Main: Mabuse.

Demenz Support Stuttgart (2010b). Wege zum Leben. Menschen mit Demenz melden sich zu Wort (DVD). Esslingen: Demenz Support Stuttgart.

Demenz Support Stuttgart (Hrsg.) (2010c). Unterstützte Selbsthilfe. In: Bausteine. Demenz. Unterstützte Selbsthilfe 06, Hannover: Vincentz.

Demenz Support Stuttgart (2012). Wir wollen mitreden! Menschen mit Demenz treten aus dem Schatten. Frankfurt/Main: Mabuse.

Filipp, S.; Aymanns, P. (2010). Kritische Lebensereignisse und Lebenskrisen. Vom Umgang mit den Schattenseiten des Lebens. Stuttgart: Kohlhammer.
Griese, B.; Griesehop, H. R. (2007). Biographische Fallarbeit. Theorie, Methode und Praxisrelevanz. Wiesbaden: VS-Verlag.

Greb, U. (2003). Identitätskritik und Lehrerbildung. Ein hochschuldidaktisches Konzept fütr die Fachdidaktik Pflege. Frankfurt/Main: Mabuse.

Goffman, E. (1975). Stigma. Über Techniken der Bewältigung beschädigter Identität. Frankfurt/ Main: Suhrkamp.

Goldsmith, M. (2005). Hearing the Voice of People with Dementia. Opportunities and Obstacles. London \& Philadelphia: Jessica Kingsley.

Hauser, U. (2006). Wenn die Vergesslichkeit noch nicht vergessen ist zur Situation Demenzkranker im frühen Stadium. Kuratorium Deutsche Altershilfe (Köln). Reihe: thema, Band 201.

Hauser, U. (2009). Wenn die Vergesslichkeit noch nicht vergessen ist. Zur Situation Demenzkranker im frühen Stadium, Köln: Kuratorium Deutsche Altershilfe.

Holzhausen, M.; Bornschlegel, U.; Fischer, T. (2009). Die Patientenperspektive in der Erfassung von Lebensqualität im Alter. Möglichkeiten und Grenzen. Zeitschrift für Gerontologie und Geriatrie, 42: 355 - 359.

Krämer, S. (2001). Sprache, Sprechakt, Kommunikation. Sprachtheoretische Positionen des 20. Jahrhunderts. Frankfurt/Main: Suhrkamp.

Kuckartz, U. (2007), Lehrbuch: Einführung in die computergestützte Analyse qualitative Daten, Wiesbaden: VS-Verlag.

Kutzleben, M.; Schmid, W.; Halek, M.; Holle, B.; Bartholomeyczik, S. (2012). Community-dwelling Persons with dementia: What do they need? What do they demand? What do they do? A systematic review of reviews on the subjective 
experiences of persons with dementia, in: Aging \& Mental Health (in press).

LaBarge, E.; Tranji, F. (1995). A Support Group for People in the Early Stages of Dementia of the Alzheimer Type. Journal of Applied Gerontology, 14, $289-301$.

Lakotta, B. (2010a). «Ein Leben wie im Fegefeuer» Der an Alzheimer erkrankte Psychologieprofessor Richard Taylor über seinen langen Weg ins Vergessen. Spiegel, 9, $111-115$

Lakotta, B. (2010b). Reise in den Sonnenuntergang. Spiegel Wissen: Die Reise ins Vergessen. Leben mit Demenz, 1, AS. 6- 17.

Lakotta, B. (2010c). «Ich bin ja noch ich». Rita Dechant, Christian Zimmermann und Helen Merlin über ihren Alltag als Demenzkranke, ihre Strategie beim Outing und ihren Umgang mit dem allmählichen Verlust der Selbständigkeit. Spiegel Wissen: Die Reise ins Vergessen. Leben mit Demenz, 1, 34-41.

Langehennig, M.; Obermann, M. (2006). Das soziale Frühstadium der Alzheimer-Krankheit. Eine kritische Wegstrecke der Krankheitsbewältigung in der Familie. Frankfurt/Main: Fachhochschulverlag.

Logsdon, R. G.; Pike, Kenneth, C.; McCurry, S. M.; Hunter, P.; Maher, J.; Snyder, L.; Teri, L. (2010). Early Stage Memory Loss Support Groups: Outcomes from a randomized controlled clinical trial, in: the journals of gerontology. Series B, Psychological Sciences and Social Sciences, 65, 691-697.

Luhmann, N. (2006). Soziale Systeme. Grundriß einer allgemeinen Theorie, Frankfurt/Main: Suhrkamp.

Niebuhr, M. (2004). Interviews mit Demenzkranken: Wünsche, Bedürfnisse und Erwartungen aus Sicht der Betroffenen. Eine qualitative Untersuchung zur subjektiven Lebens- qualität von Demenzkranken. Köln: Kuratorium Deutsche Altershilfe.

Panke-Kochinke, B. (2004). Die rekonstruktive hermeneutische Textanalyse. Pflege und Gesellschaft, 2, 59-63.

Panke-Kochinke, B. (2008). Gewalt gegen Pflegekräfte. Problematische Situationen erkennen und lösen, Frankfurt/Main: Mabuse.

Panke-Kochinke, B. (2012). Menschen mit Demenz im Frühstadium ihrer Erkrankung - wie kann man ihre Selbstbestimmung fördern? Bausteine für eine Konzeptentwicklung in der Versorgungsforschung, Pflege \& Gesellschaft, 2, $162-176$.

Pasero, U.; Backes, G. M.; Schroeter, K. R. (Hrsg.) (2007). Altern in Gesellschaft. Ageing - Diversity - Inclusion, Wiesbaden: VS-Verlag.

Roest, H. G. van der, Meiland, F. J. M. Marrocine, R., et. Al. (2007). Subjective needs of people with dementia: a review of the literature. International Psychogeriatrics, 19, 3, $559-592$.

Rohra, H. (2011), Aus dem Schatten treten, Frankfurt/Main: Mabuse.

Rosenthal, G. (1995). Erlebte und erzählte Lebensgeschichte, Frankfurt/Main: CAMPUS Verlag.

Rosenthal, G. (2010). Interpretative Sozialforschung. Eine Einführung, Weinheim \& München: Juventa Verlag.

Schütze, F. (1981). Prozeßstrukturen des Lebenslaufs, in: Matties, J.; Pfeifenberger, A.; Stosberg, M, Biographie in handlungswissenschaftlicher Perspektive. Forschungsvereinigung Nürnberg, 67 - 156.

Schütze, F. (1983). Biographieforschung und narratives Interview, Neue Praxis, 13, 283 - 293.

Snyder, L.; Jenkins, C.; Joosten, L. (2007). Effectiveness of Support Groups for People with Mild to Moderate Alzheimer's Disease: An Evaluative Survey. American Journal of Alz- heimer's Disease and Other Dementias, 22, 14- 19.

Snyder, L. (2009). Speaking Our Minds. What it's like to have Alzheimer's. Baltimore: Health Professions Press.

Sowarka, D. (2008). Demenz im Frühstadium. Forschung zur Betroffenenperspektive und Implikation für Behandlung und Begleitung, Informationsdienst Altersfragen, 06, 1-6.

Stechl, E. (2006a). Subjektive Wahrnehmung und Bewältigung der Demenz im Frühstadium. Eine qualitative Interviewstudie mit Betroffenen und ihren Angehörigen. Berlin: Verlag Dr. Köster.

Stechl, E.; Lämmler, G.; SteinhagenThiessen, E.; Flick, U. (2006b). Subjektive Wahrnehmung und Bewältigung der Demenzim Frühstadium - SUWADEM. Eine qualitative Interviewstudie mit Betroffenen und ihren Angehörigen. In: Demenz eine Herausforderung für das 21. Jahrhundert. 100 Jahre AlzheimerKrankheit (Tagungsreihe der Deutschen Alzheimer Gesellschaft e.V., Bd. 6), 223-229.

Stechl, E.; Lämmler, G.; SteinhagenThiessen, E.; Flick, U. (2007). Subjektive Wahrnehmung und Bewältigung der Demenzim Frühstadium - SUWADEM. Eine qualitative Interviewstudie mit Betroffenen und Angehörigen. Zeitschrift für Gerontologie und Geriatrie, 40, $71-80$.

Steeman E, de Casterlé BD, Godderis J, Grypdonck M. (2006), Living with early-stage dementia: a review of qualitative studies. Journal of Advanced Nursing, 54, $722-738$.

Yale, R. (1995). Developing Support Groups for Individuals with EarlyStage Alzheimer's Disease. Planning, Implementation, and Evaluation, Baltimore: Health Professions Press. 
Was war die größte Herausforderung bei Ihrer Studie?

Die größte positive Herausforderung der Studie bestand darin, zu erschließen, wie Menschen mit Demenz, die sich in einer Selbsthilfegruppe engagieren, die Bewältigung der Demenz selbst beschreiben.

Was wünschen Sie sich bezüglich der Thematik für die Zukunft?

Selbsthilfegruppen für Menschen mit Demenz ergänzen das Unterstützungsangebot in der Form, dass sie auch für jeden Betroffenen und seine Angehörigen in seinem näheren Wohnumfeld ohne Schwierigkeiten zu erreichen sind.

Was empfehlen Sie den LeserInnen zum Weiterlesen/Vertiefen? Alle Publikationen, in denen Menschen mit Demenz ihre Bedürfnisse und Bedarfe selbst darstellen (z. B. Rohra, Zimmermann, Bryden, Taylor etc.) empfehlen sich zum Weiterlesen.

Zur Vertiefung empfiehlt sich Stechl, E. (2006). Subjektive Wahrnehmung und Bewältigung der Demenz im Frühstadium. Eine qualitative Interviewstudie mit Betroffenen und ihren Angehörigen, Berlin: Verlag Dr. Köster.

\section{Korrespondenzadresse}

Privatdozentin

Dr. Birgit Panke-Kochinke Wissenschaftliche Mitarbeiterin Deutsches Zentrum für Neurodegenerative Erkrankungen Standort Witten

Postfach 6250
Stockumer Straße 12 58453 Witten

Deutschland

Tel.: +49 2302 926-227

Fax: +49 2302 926-239

birgit.panke-kochinke@dzne.de 